\title{
Combined usage of Wilms' tumor gene quantitative analysis and multiparameter flow cytometry for minimal residual disease monitoring of acute myeloid leukemia patients after allogeneic hematopoietic stem cells transplantation
}

\author{
YINGCHAN HAO, YANHONG CHENG, QUAN WU, AIMEI ZHANG, XIAOXIAO JIANG and XIUCAI XU \\ Central Laboratory, The Affiliated Provincial Hospital, Anhui Medical University, Hefei, Anhui 230001, P.R. China
}

Received December 15, 2016; Accepted July 7, 2017

DOI: $10.3892 /$ etm.2017.5547

\begin{abstract}
High expression of the Wilms' tumor gene (WT1) in acute myeloid leukemia (AML) has been considered as a sensitive marker of minimal residual disease (MRD). The present study investigated the significance of quantitative analysis of WT1 mRNA, combined with multiparameter flow cytometry (MFC) regarding its efficacy and prognostic as well as relapse prediction value for leukemia patients with hematopoietic stem cell transplantation. Reverse-transcription quantitative polymerase chain reaction analysis demonstrated that the expression of WT1 in the initial and relapse group was significant higher than that in the complete remission (CR) group $(\mathrm{P}<0.01)$. WT1 and the donor chimerism were negatively correlated $(\mathrm{r}=-0.73, \mathrm{P}<0.05)$. In all AML patients, WT1 was the highest in the M3 subtype and the lowest in the M1 subtype. Follow-up of 12 AML patients demonstrated that WT1 gene expression levels markedly decreased after $\mathrm{CR}$, but obviously increased after relapse, as did the rate of the leukemia cells detected by MFC. The combined usage of MFC and WT1 monitoring contributed to an improved detection rate of relapse (91.7\%), and may be used to monitor MRD, assess the treatment efficacy and prognosis, and predict the risk of recurrence in leukemia patients without specific molecular markers after allogeneic hematopoietic stem cell transplantation.
\end{abstract}

\section{Introduction}

Acute myeloid leukemia (AML) is a clinically and biologically heterogeneous malignancy involving hematopoietic

Correspondence to: Professor Xiucai Xu, Central Laboratory, The Affiliated Provincial Hospital, Anhui Medical University, 17 Lujiang Road, Hefei, Anhui 230001, P.R. China

E-mail: xuxiucai1972@163.com

Key words: Wilms' tumor gene, acute myeloid leukemia, reverse-transcription quantitative polymerase chain reaction, multiparameter flow cytometry, minimal residual disease stem cells (HSCs) and progenitor cells which lost their normal ability to proliferate, differentiate and mature, while continuously proliferating. Allogeneic HSC transplantation (HSCT) is currently deemed the most effective treatment approach for AML. Despite the high remission rate, a significant proportion of patients with AML experience a relapse. Thus, precise evaluation of minimal residual disease (MRD) after allogeneic HSCT is particularly important for assessment of the therapeutic efficacy (1). Various approaches have been used to define MRD: Chromosome karyotype analysis, reverse-transcription quantitative polymerase chain reaction (RT-qPCR) (2,3), multiparameter flow cytometry (MFC) $(4,5)$ and fluorescence in situ hybridization (FISH) (6). RT-qPCR has a higher sensitivity compared to the aforementioned techniques as target mRNAs can be detected even when diluted to 1 in $10^{4}-10^{5}$ or even 1 in $10^{6}$ (2). However, several studies have confirmed that only a portion of AML patients harbored specific genetic markers, which produced certain obstacles for monitoring MRD. Therefore, there is an imperative need to develop alternative markers for MRD analysis in a greater proportion of patients.

The transcript of the Wilms' tumor gene (WT1) is detectable in the bone marrow (BM) of $90 \%$ of patients with AML by RT-qPCR (7). WT1 encodes a zinc finger transcription factor with a complex pattern of alternative splicing with different binding specificities locating at chromosome 11p13. Compared with normal BM cells, WT1 mRNA is highly expressed in the BM of patients with different types of leukemia, particularly AML. Furthermore, it has been confirmed that the WT1 mRNA levels are closely correlated with the disease status. Hence, the value of WT1 detection in monitoring leukemia treatment efficacy is of high significance (8).

The aim of the present study was to explore whether the mRNA levels of WT1 are an effective marker for MRD in AML after allogeneic HSCT. The predictive value of the combined usage of WT1 quantification, MRD monitoring by flow cytometry (FCM) and assessment of donor chimerism (DC) by short tandem repeats (STR)-PCR regarding treatment efficacy and prognosis, as well as the capacity to predict the risk of recurrence of AML after potentially curative treatment by HSCT, were evaluated. 


\section{Materials and methods}

Patients. A total of 72 AML patients (34 men and 38 women) who were schedules to undergo allogeneic HSCT at the Affiliated Provincial Hospital of Anhui Medical University (Hefei, China) between March 2013 and July 2015 were enrolled in the present study. The median age of the patients was 43 years (range, 1-70 years). A total of $72 \mathrm{BM}$ samples and $\mathrm{PB}$ samples were collected during diagnosis and follow-up in sterile tubes with EDTA anti-coagulant. Of the 72 patients diagnosed with AML, 60 (83.3\%) achieved complete remission (CR), while $12(16.7 \%)$ experienced a relapse after allogeneic HSCT. In addition, 22 cases of non-malignant blood disease, including iron deficiency anemia, immune thrombocytopenic purpura, allergic purpura or gigantic young cell anemia were enrolled as a control group. The diagnosis of the patients was based on the French-American-British diagnostic criteria for AML and confirmed by morphology, immunophenotyping, cytogenetics and molecular biology (9). The contents of all experiments were approved by the medical ethics committee of the Affiliated Provincial Hospital of Anhui Medical University (Hefei, China) and informed consent was obtained from all patients or their guardians (Table I).

Quantification of WT1. BM mononuclear cells were separated by a Ficoll-Hypaque solution then centrifuged at $400 \mathrm{x} g$ at $4^{\circ} \mathrm{C}$ for $20 \mathrm{~min}$. The total RNA was extracted using TRIzol reagent (Invitrogen; Thermo Fisher Scientific, Inc., Waltham, MA, USA) according to the manufacturer's protocol within $24 \mathrm{~h}$ of sample collection. The concentration of the extracted RNA was evaluated by a spectrophotometer. A total of $15 \mu \mathrm{l}$ RNA $(\sim 1 \mu \mathrm{g})$ was used for one-step RT-qPCR WT1 kit (cat. no. CA000022M; Shanghai Yuanqi Bio-Pharmaceutical Co., Ltd., Shanghai, China); this kit also contained the primers for WT1 and ABL. The preparation of reaction mixtures was according to the manufacturer's protocol, and reactions were performed in an ABI 7500 real-time PCR system (Thermo Fisher Scientific, Inc.). One step RT-qPCR was performed as follows: $42^{\circ} \mathrm{C}$ for $30 \mathrm{~min}$, initial denaturation at $94^{\circ} \mathrm{C}$ for $5 \mathrm{~min}$, and 40 cycles of denaturation with $94^{\circ} \mathrm{C}$ for $15 \mathrm{sec}$ and annealing at $60^{\circ} \mathrm{C}$ for $1 \mathrm{~min}$. The levels of the WT1 transcript were expressed as the number of WT1 copies per 100 copies of the Abelson gene (ABL) (10).

MFC. BM samples $\left(100 \mu 1 ; 1 \times 10^{6}\right.$ nucleated cells) were incubated with the following antibodies in a 1:5 dilution: CD45 (cat. no. 560777), CD11B (cat. no. 560481) (both BD Biosciences, San Jose, CA, USA), CD19 (cat. no. 302216; BioLegend, Inc., San Diego, CA, USA), CD56 (cat. no. 555518), CD13 (cat. no. 557454), HLA-DR (cat. no. 335796) and CD34 (cat. no. 347203) (all BD Pharminogen; BD Biosciences), CD117 (cat no. IM2732U), CD15 (cat. no. IM1423U) and CD7 (cat. no. A07755) (all Beckman Coulter, Inc., Brea, CA, USA). These antibodies were incubated in two combinations: CD7-FITC/CD117-PE/CD34-Percp-cy5.5/CD13-APC and CD15-FITC/CD117-PE/CD34-Percp-cy5.5/CD56-APC/CD19PE-CY7/CD11B-Pacific Blue/HLA-DR-APC-Cy7/CD45Amcyan. Red blood cells were lysed by Lysing Solution 10X Concentrate (BD Biosciences) at $37^{\circ} \mathrm{C}$ for $15 \mathrm{~min}$, followed
Table I. Study population characteristics.

\begin{tabular}{lc}
\hline Characteristic & Value \\
\hline Number of patients & 72 \\
Males/females & $34 / 38$ \\
Age at diagnosis (years) & $43(1-70)$ \\
FAB subtype & \\
M0 & 3 \\
M1 & 2 \\
M2 & 20 \\
M3 & 25 \\
M4 & 10 \\
M5 & 8 \\
M6 & 4 \\
Median time of observation & $12(1-89)$ \\
after allogeneic HSCT (months) & \\
Status & 12 \\
Relapse & 60 \\
CR & 22 \\
Number of controls & \\
\hline Values are expressed as $n$ or as median (range). FAB, French- \\
American-British classification; CR, complete remission; HSCT, \\
hematopoietic stem cell transplantation; M, FAB classification of \\
acute myeloid leukemia subtypes.
\end{tabular}

by centrifugation at $116 \mathrm{xg}$ at $20^{\circ} \mathrm{C}$ for $5 \mathrm{~min}$. The cells were washed with PBS and then analyzed on a BD FACSCalibur flow cytometer (BD Biosciences) and the data were processed by Diva 6.1 software (BD Biosciences). A total of $1.0 \times 10^{5}$ cells were analysed from each tube, with 20,000 events acquired for patients with MRD or relapse. Blasts were identified by using a CD45/side scatter log gating strategy. The selection of the monoclonal antibodies was based on leukemia antigen profiles of the original diagnostic specimen. Samples with $>0.01 \%$ leukemic cells in BM were defined as positive; otherwise, they were defined as negative. Detection of $>5 \%$ leukemia cells in BM by morphological observation or FCM was considered to indicate a relapse.

STR-PCR. STR-PCR was used to assess DC. According to the manufacturer's protocol and the instructions of the ABIPRISM 3100 Genetic Analyzer (Thermo Fisher Scientific, Inc.), DC was detected in all patients receiving HSCT. Detection was performed at days 7,14 and 21 after transplantation and in patients with recurrence, and the results were analysed using GeneMapper ID-X software (version 1.2; Thermo Fisher Scientific, Inc.). According to the effective site of peak area percentage, quantitative calculation of donor cells was performed using the following formula: $\mathrm{DC}$ ratio $=$ donor area/(donor area + recipient area).

Statistical analysis. Results were analysed by using SPSS 17.0 software (SPSS Inc., Chicago, IL, USA). For WT1 mRNA levels, comparison between two groups was performed using the independent t-test. The log-rank tests were drawn 
Table II. Expression of WT1 mRNA and DC in patients with acute myeloid leukemia at different time-points after allogeneic hematopoietic stem cell transplantation.

\begin{tabular}{lccccccc}
\hline Time-point/ group & $\mathrm{n}$ & WT1 $(\%$ of ABL $)$ & $\mathrm{t}$ & P-value $^{\mathrm{a}}$ & DC ratio $(\%$ abnormal cells $)$ & $\mathrm{t}$ & P-value $^{\mathrm{a}}$ \\
\hline 7 days & 12 & $3.58 \pm 1.19$ & 2.83 & $<0.01$ & $50.25 \pm 18.59$ & 4.68 & $<0.01$ \\
14 days & 12 & $1.18 \pm 0.65$ & 6.82 & $<0.01$ & $94.63 \pm 4.20$ & 9.47 & $<0.01$ \\
21 days & 12 & $0.24 \pm 0.08$ & 8.64 & $<0.01$ & $97.53 \pm 1.33$ & 13.64 & $<0.01$ \\
Relapse group & 12 & $5.52 \pm 2.14$ & - & - & $76.32 \pm 5.22$ & - & - \\
\hline
\end{tabular}

${ }^{a}$ Compared with the relapse group. Correlation coefficient for WT1 vs. DC for all groups, $\mathrm{r}=-0.73$. As the DC ratio is only analysed after hematopoietic stem cell transplantation surgery and recipients did not have donor cells their bodies at time-point 0 , the DC ratio detection is not conducted in routine clinical practice at time-poiunt 0. WT1, Wilms' tumor gene; ABL, Abelson gene; DC, donor chimerism.
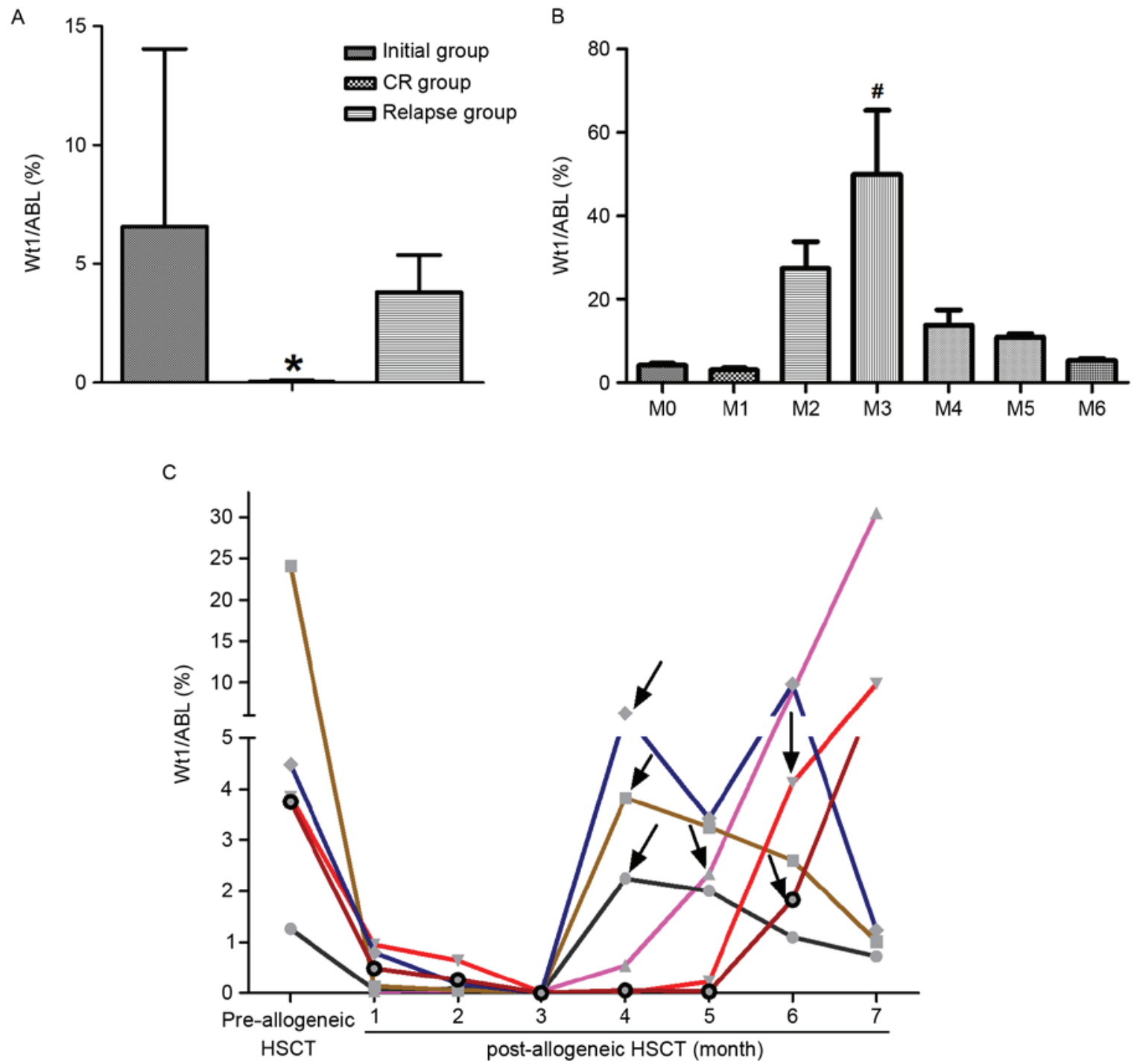

Figure 1. WT1 mRNA levels of in AML patients pre- and post-allogeneic HSCT. (A) Expression levels of WT1 in AML patients from three groups. (B) WT1 expression levels in AML of variable AML subtypes M1-M6 according to the French-American-British classification. (C) Dynamic monitoring of WT1 expression levels in 6 relapse patients pre- and post-allogeneic HSCT. Black arrows indicate clinical recurrence. ${ }^{*} \mathrm{P}<0.01$, ${ }^{*} \mathrm{P}<0.05$ compared with other groups. WT1/ABL, Wilms' tumor gene/Abelson; CR, complete remission; HSCT, hematopoietic stem cell transplantation; AML, acute myeloid leukemia; M, French-American-British classification of AML subtypes.

to determine the survival probabilities of patients based on their WT1 status and was used for comparing patients with or without MRD. Furthermore, a negative correlation was found between WT1 and DC through Pearson's correlation analysis. $\mathrm{P}<0.05$ was considered to indicate a statistically significant difference between groups. 


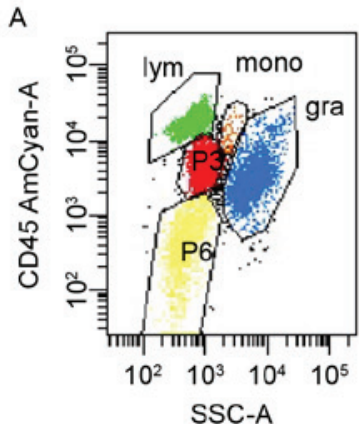

B

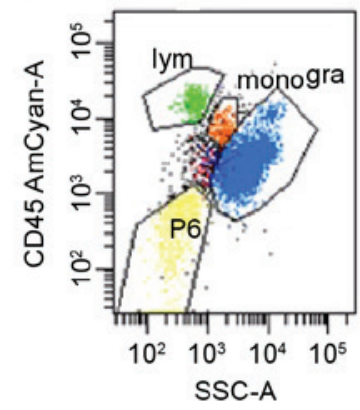

C

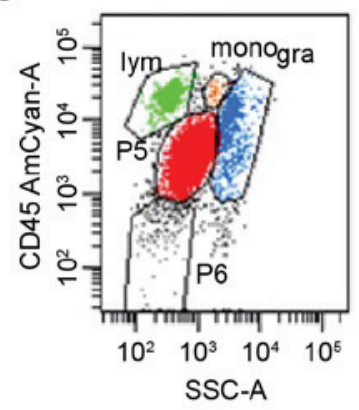

D

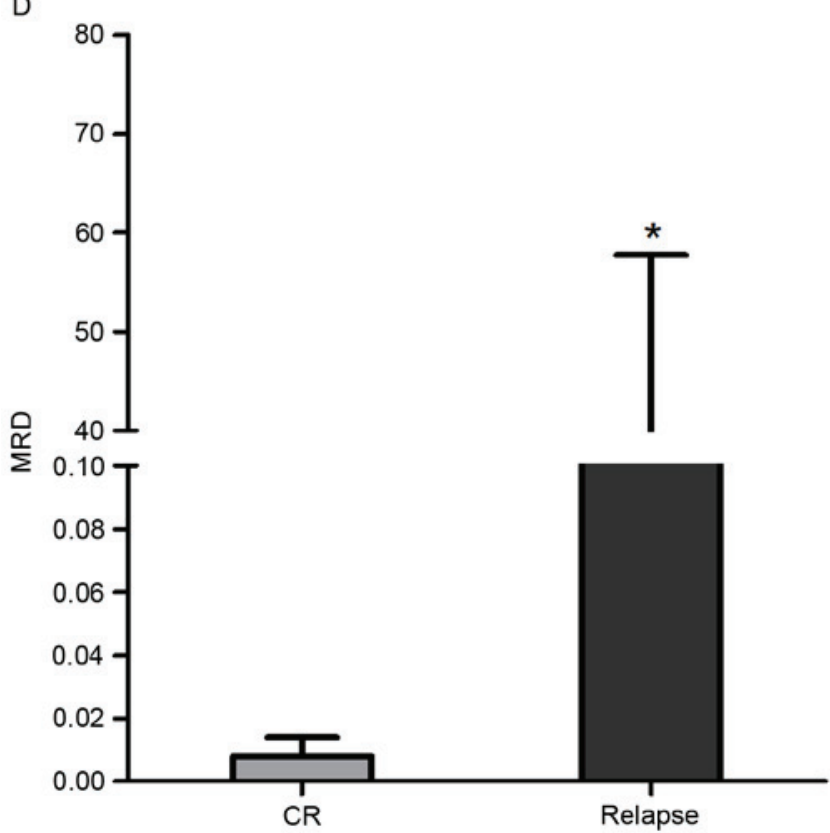

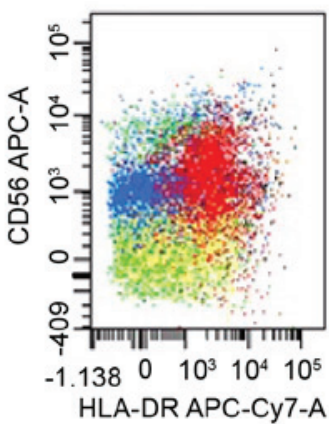
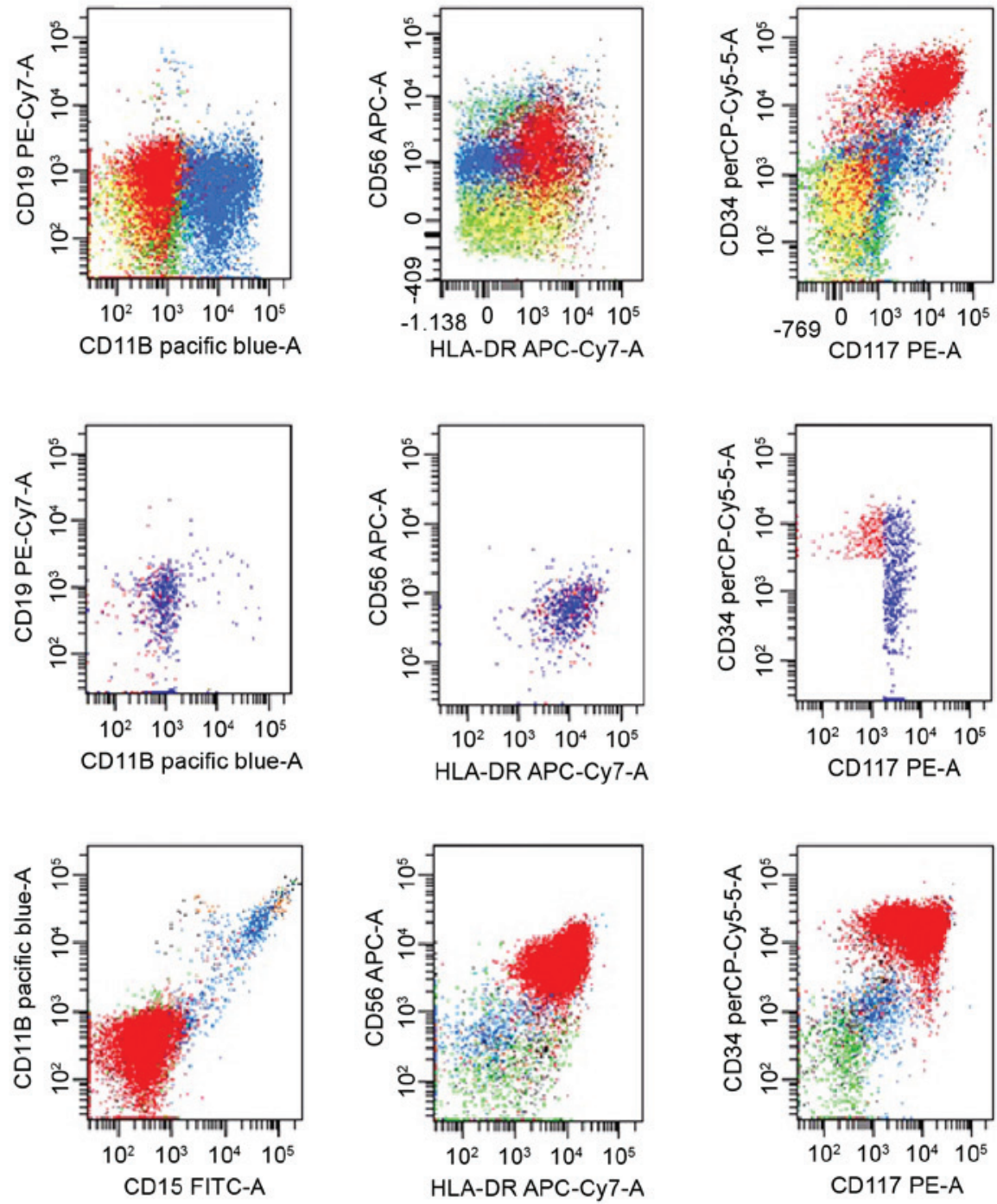

Figure 2. MRD detection in consecutive BM samples of (A) a patient in the initial group, (B) a patient in the CR group and (C) a relapsing patient (abnormal cells marked in red). As the subjects in the present study were CR patients, the percentage of abnormal leukemia cells was low. Therefore abnormal leukemia cells can only be shown as gated populations. (D) MRD levels (percentage of abnormal cells) in the CR group and the relapse group. "P<0.01, compared with the CR group. MRD, minimal residual disease; CR, complete remission; SSC, side scatter; lym, lymphocytes; mono, monocytes; gra, granulocytes; FITC, fluorescein isothiocyanate; PE, phycoerythrin; APC, allophycocyanin; BM, bone marrow; HLA-DR, human leukocyte antigen - antigen D related; PerCP, peridinin chlorophyll. 

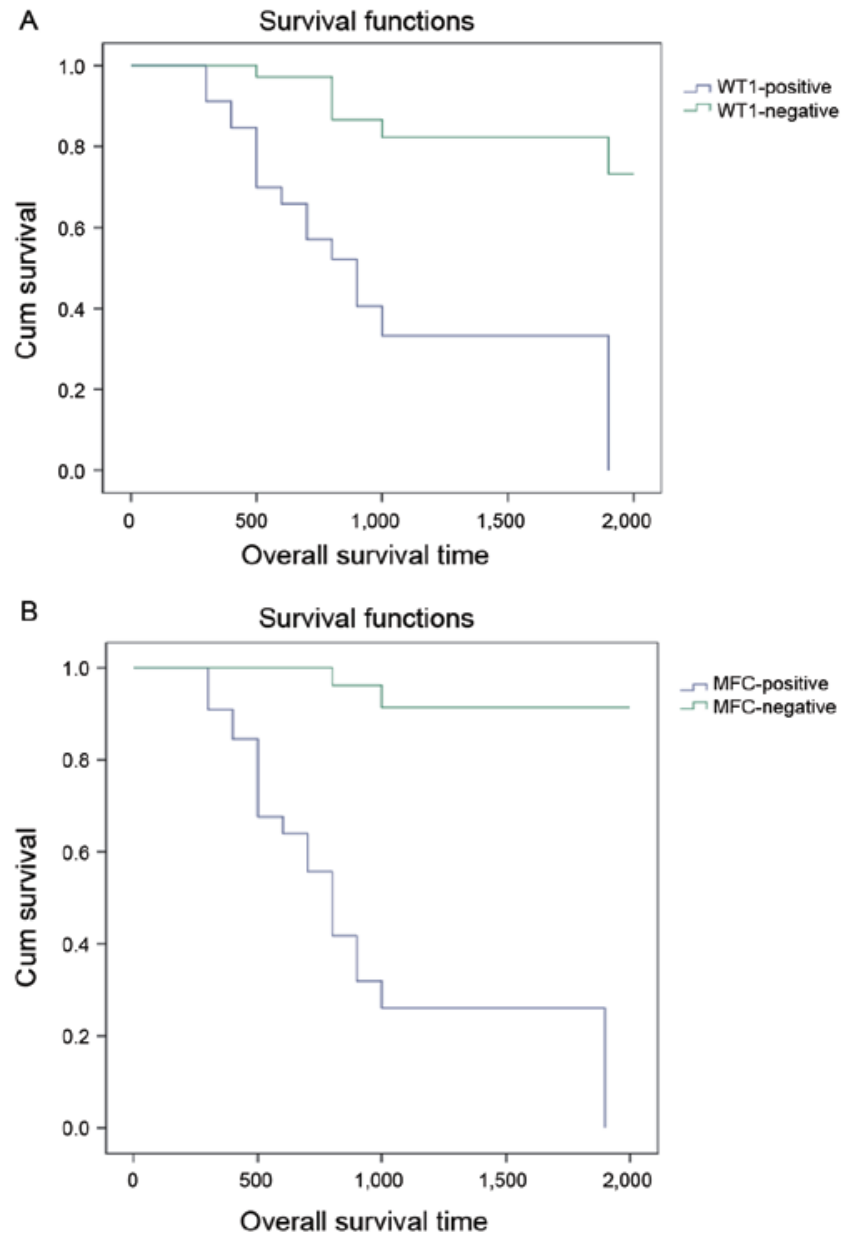

Figure 3. Kaplan-Meier overall survival curves for patients stratified by (A) WT1 status determined by reverse-transcription quantitative polymerase chain reaction analysis and (B) minimal residual disease detected by MFC with the survival time stated in days $(\mathrm{P}<0.01$ for each). Cum, cumulative; WT1, Wilms' tumor gene; MFC, multiparameter flow cytometry.

\section{Results}

WT1 mRNA levels in AML patients pre- and post-allogeneic $H S C T$. RT-qPCR demonstrated that the expression of WT1 in AML patients at baseline was significantly higher than in those in relapse and $\mathrm{CR}$ groups $(\mathrm{P}<0.01$, respectively). Compared with that in the CR group, WT1 in the AML relapse group was significantly increased $(\mathrm{P}<0.01$; Fig. 1A).

The differences of WT1 mRNA levels at different time-points after allogeneic HSCT were statistically significant $(\mathrm{P}<0.01)$. Pairwise comparison indicated that the expression of WT1 in the relapse group was higher than that at 7,14 and 21 days post-transplantation $(\mathrm{P}<0.01$, respectively). However, DC in the relapse group was significantly lower than that in the groups at 14 and 21 days. Through correlation analysis, a negative correlation between WT1 and DC was identified $(\mathrm{r}=-0.73, \mathrm{P}<0.05$; Table II).

As indicated in Fig. 1B, among all newly diagnosed AMLs, the highest WT1 levels were found in the M3 and the lowest in the M1 subtype. A significant difference was found between $\mathrm{M} 3$ and the other subtypes $(\mathrm{P}<0.05)$.

In addition, monitoring the WT1 expression levels of 12 AML patients with a relapse by RT-qPCR indicated that WT1

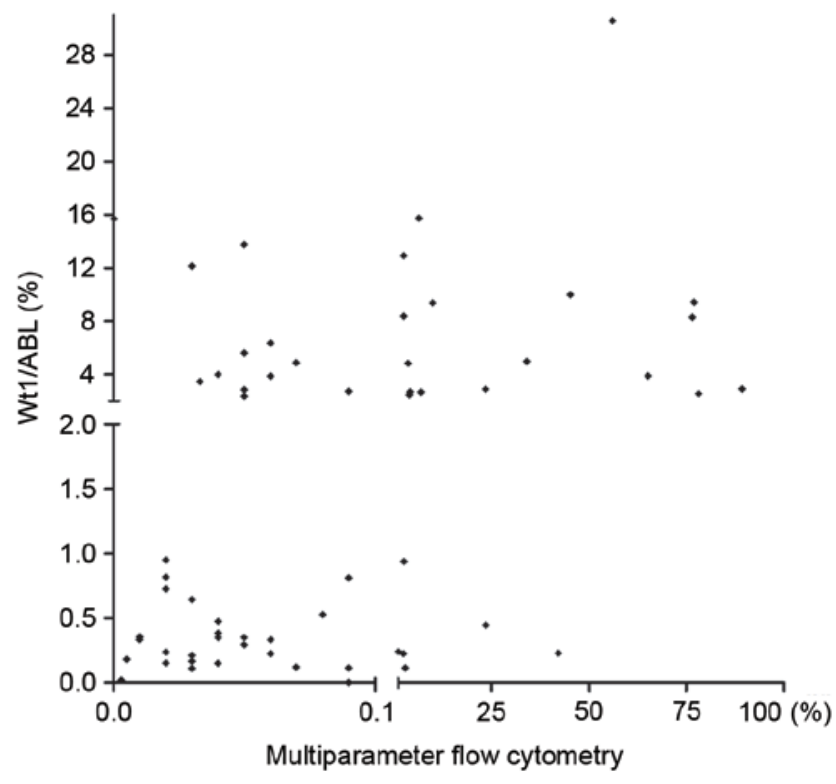

Figure 4. The association between of WT1/ABL mRNA and MFC in MRD detection. Each data-point represents the measurements for one sample. Patients with WT1/ABL mRNA levels of $>2$ and $<0.1 \%$ leukemia cells in bone marrow according to MFC were considered to have MRD. WT1/ABL, Wilms' tumor gene/Abelson; MFC, multiparameter flow cytometry; MRD, minimal residual disease.

gene expression levels were high at baseline and then markedly decreased after $\mathrm{CR}$, but obviously increased after the relapse. Dynamic monitoring over seven consecutive months revealed that WT1 levels significantly increased prior to clinical recurrence (Fig. 1C).

Monitoring MRD by MFC pre- and post-allogeneic HSCT. The present study used a cutoff value for MRD of $0.1 \%$ leukemic cells in the BM. The MFC results indicated that certain patients with average leukemic cell percentages in the $\mathrm{BM}$ of $<0.01 \%$ were still in $\mathrm{CR}$, while those with percentages of $\geq 2.0 \%$ developed a relapse. Relatively small amounts of MRD were cleared by further induction chemotherapy courses. In patients with AML after allogeneic HSCT, the presence of MRD determined by MFC prompts risk-adopted therapy to prevent recurrence. A representative patient whose MFC results are presented in Fig. 2 was diagnosed as M2a (Fig. 2A). After allogeneic HSCT, the percentage of leukemic cells in BM decreased to $0 \%$ (Fig. 2B), while it markedly increased when the patient experienced a relapse of AML (84.9\%; Fig. 2C). In the present study, the percentage of leukemic cells in BM in all AML patients in CR was $0.008 \pm 0.006 \%$ and was significantly increased in the relapse group (35.5 $\pm 22.3 \%$; Fig. 2D).

Overall survival (OS). The log-rank test revealed significant differences in OS between patients stratified by the presence or absence of MRD as determined by their WT1 status and the MFC results. Patients were regarded to have a negative status if the initially positive WT1 status or MFC results turned negative (WT1/ABL mRNA levels of $<2$ and $<0.1 \%$ leukemic cells in BM as detected by MFC, respectively) at a certain point during the observation period. Median OS was 2,000 days in the group of patients who were WT1-negative and 818 days 
for those who were positive $(\mathrm{P}<0.01)$. In the group of patients who had no MRD according to MFC, the median OS was 1,900 days, while that in patients with MRD was 741 days $(\mathrm{P}<0.01$; Fig. 3)

Association of MRD determined by MFC and via WT1 mRNA determined by $R T-q P C R$. The WT1 mRNA levels determined by RT-qPCR were compared with the presence of MRD determined by MFC in BM specimens obtained from each of the 72 patients (Fig. 4). Of these, 12 (16.7\%) were positive for WT1 mRNA but had no MRD according to MFC, and 9 (12.5\%) had MRD according to MFC but were negative for WT1 mRNA. A total of 22 samples (30.6\%) were double-negative, while 29 samples $(40.3 \%)$ were positive according to MFC as well as RT-qPCR of WT1. In the population of AML patients, there was no significant difference in MRD determined via WT1 by RT-qPCR and that determined by MFC. These results indicated that, regarding the determination of MRD, the sensitivity of MFC is comparable to that of RT-qPCR analysis of WT1 mRNA. Of note, among the 12 relapse patients, $11(91.7 \%)$ were double-positive. Therefore, combined use of MRD monitoring via WT1 and MFC may increase the sensitivity of predicting relapse after allogeneic HSCT without affecting the specificity.

\section{Discussion}

Highly sensitive and efficient monitoring of MRD is crucial for patients with AML receiving allogeneic HSCT (11). RT-qPCR and MFC have been confirmed to be sufficiently sensitive and specific to serve as standard approaches for MRD monitoring in AML. While RT-qPCR detection may reach a higher sensitivity $\left(10^{-5}-10^{-6}\right)$ than other methods, including FISH and MFC (12), its applicability was traditionally restricted to those patients harbouring specific fusion genes, such as promyelocytic leukemia-retionic acid receptor a, AML1-ETO, TEL-AML1 and breakpoint cluster region-ABL. It is known that $>60 \%$ of AML patients lack specific molecular targets, and it is therefore important to identify alternative molecular markers applicable for the majority of AML patients (13). Retrospective analysis of cohorts of previous studies identified that quantification of WT1 mRNA in BM specimens may be used as a marker of MRD and predict a relapse of AML after allogeneic HSCT $(14,15)$. Marjanovic et al $(16)$ confirmed that WT1 transcript levels were associated with clinical remission and relapse. In addition, several studies proved that the combination of quantification of WT1 and MFC increased the sensitivity of MRD analysis $(17,18)$.

In the present study, statistical analyses were used to assess the predictive value of MRD standards regarding mortality after allogeneic HSCT. The cutoff values for MRD determined by MFC and via WT1 by RT-qPCR were identified as 0.1 and $2.0 \%$ ABL, respectively, according to previously established protocols (19). Consistent with this study, the present study identified a close association between WT1 gene expression and disease status after allogeneic HSCT. AML patients with overexpression of WT1 mRNA at disease onset were enrolled in the present study. The expression of WT1 in the AML relapse group was significantly higher than that in the CR group (20). To further demonstrate the value of WT1 as a predictor of relapse, the present study detected WT1 mRNA expression in BM specimens from patients with allogeneic HSCT at the scheduled time-points (every 7 days in the first month and subsequently once a month until relapse). In those patients, overexpression of WT1 was detected immediately prior to a relapse by regular monthly follow-up after allogeneic HSCT. Given the negative correlation between WT1 and the donor gene chimeric rate, the quantification of WT1 was an effective complement to the donor gene chimeric rate as a warning signal of transplant rejection (21). From the data of 12 AML patients who suffered a relapse, an association of high levels of abnormal leukaemia cells according to MFC after allogeneic HSCT with an lower OS was identified. MFC had a higher sensitivity but a lower specificity than WT1 detection at each time-point. The results of the present study indicated the high expression of WT1 levels can predict disease recurrence: The WT1 mRNA status $(\mathrm{P}<0.01)$ as well as MRD determined by MFC $(\mathrm{P}<0.01)$ was associated with $\mathrm{OS}$ and had a prognostic value. Almost all relapse cases were double-positive for WT1 and MFC results. Therefore, the combination of MFC and WT1 mRNA quantification may be used for monitoring MRD in AML without any specific molecular targets to assess the treatment efficacy and prognosis, and predict the risk of recurrence in AML patients. Several studies have identified that high levels of WT1 mRNA prior to allogeneic HSCT may be correlated with poor outcome of AML after HSCT (22). Future research performed by our group will assess more typical cases and further confirm the conclusions of the present study, combined with other relevant laboratory indexes based on certain in vitro experiments. Further work should also further evaluate the accurate predictive value of MRD determined by WT1 mRNA detection and MFC (23).

In conclusion, WT1 may be a sensitive marker for predicting OS of AML in patients receiving allogeneic HSCT. The present study confirmed that monitoring of the MRD status by MFC and quantitative expression of the WT1 gene prior to and following allogeneic HSCT had a significant influence on AML patients without any specific molecular targets. An additional study indicated that WT1 mRNA quantification and MFC had a comparable ability to identify high-risk patients who eventually relapsed. Furthermore, the combination of WT1 mRNA detection and MFC markedly increased the sensitivity of predicting the risk of recurrence of AML after allogeneic HSCT (24). Future research will verify these results and explore more appropriate markers for this purpose.

\section{Acknowledgements}

This study was supported by the Anhui Province Natural Science Foundation (grant no. 1708085MH223).

\section{References}

1. Malagola M, Skert C, Borlenghi E, Chiarini M, Cattaneo C, Morello E, Cancelli V, Cattina F, Cerqui E, Pagani C, et al: Postremission sequential monitoring of minimal residual disease by WT1 Q-PCR and multiparametric flow cytometry assessment predicts relapse and may help to address risk-adapted therapy in acute myeloid leukemia patients. Cancer Med 5: 265-274, 2016.

2. Elmaagacli AH: Molecular methods used for detection of minimal residual disease following hematopoietic stem cell transplantation in myeloid disorders. Methods Mol Biol 1109: 187-207, 2014. 
3. Yamauchi T, Negoro E, Lee S, Takai M, Matsuda Y, Takagi K, Kishi S, Tai K, Hosono N, Tasaki T, et al: Detectable Wilms' tumor-1 transcription at treatment completion is associated with poor prognosis of acute myeloid leukemia: A single institution's experience. Anticancer Res 33: 3335-3340, 2013.

4. Fossat C, Roussel M, Arnoux I, Asnafi V, Brouzes C, Garnache-Ottou F, Jacob MC, Kuhlein E, Macintyre-Davi E, Plesa A, et al: Methodological aspects of minimal residual disease assessment by flow cytometry in acute lymphoblastic leukemia: A French multicenter study. Cytometry. Part B, Clin Cytomet 88: 21-29, 2015.

5. Ikoma MR, Beltrame MP, Ferreira SI, Souto EX, Malvezzi M and Yamamoto M; Minimal ResidualDisease Working Group of the Brazilian Society of Bone Marrow Transplantation (SBTMO): Proposal for the standardization of flow cytometry protocols to detect minimal residual disease in acute lymphoblastic leukemia. Rev Bras Hematol Hemoter 37: 406-413, 2015.

6. Rossi G, Minervini MM, Carella AM, de Waure C, di Nardo F, Melillo L, D'Arena G, Zini G and Cascavilla N: Comparison between multiparameter flow cytometry and WT1-RNA quantification in monitoring minimal residual disease in acute myeloid leukemia without specific molecular targets. Leuk Res 36 401-406, 2012.

7. Rezai O, Khodadadi A, Heike Y, Mostafai A, Gerdabi ND, Rashno M and Abdoli Z: Assessment of relationship between Wilms' Tumor Gene (WT1) expression in peripheral blood of acute leukemia patients and serum IL-12 and C3 levels. Asian Pac J Cancer Prev 16: 7303-7307, 2015.

8. Zhang R, Yang JY, Sun HQ, Jia H, Liao J, Shi YJ and Li G: Comparison of minimal residual disease (MRD) monitoring by WT1 quantification between childhood acute myeloid leukemia and acute lymphoblastic leukemia. Eur Rev Med Pharmacol Sci 19: 2679-2688, 2015.

9. Vardiman JW, Thiele J, Arber DA, Brunning RD, Borowitz MJ, Porwit A, Harris NL, Le Beau MM, Hellström-Lindberg E, Tefferi A and Bloomfield CD: The 2008 revision of the World Health Organization (WHO) classification of myeloid neoplasms and acute leukemia: Rationale and important changes. Blood 114: 937-951, 2009

10. Livak KJ and Schmittgen TD: Analysis of relative gene expression data using real-time quantitative PCR and the 2(-Delta Delta C(T)) method. Methods 25: 402-408, 2001.

11. Niavarani A, Herold T, Reyal Y, Sauerland MC, Buchner T, Hiddemann W, Bohlander SK, Valk PJ and Bonnet D: A 4-gene expression score associated with high levels of Wilms Tumor-1 (WT1) expression is an adverse prognostic factor in acute myeloid leukaemia. Br J Haematol 172: 401-411, 2016.

12. Willasch AM, Gruhn B, Coliva T, Kalinova M, Schneider G, Kreyenberg H, Steinbach D, Weber G, Hollink IH, Zwaan CM, et al: Standardization of WT1 mRNA quantitation for minimal residual disease monitoring in childhood AML and implications of WT1 gene mutations: A European multicenter study. Leukemia 23: 1472-1479, 2009.

13. de Haar C, Plantinga M, Blokland NJ, van Til NP, Flinsenberg TW, Van Tendeloo VF, Smits EL, Boon L, Spel L, Boes M, et al: Generation of a cord blood-derived Wilms Tumor 1 dendritic cell vaccine for AML patients treated with allogeneic cord blood transplantation. Oncoimmunology 4: e1023973, 2015.
14. Sakamoto Y, Mariya Y, Sasaki S, Teshiromori R, Oshikiri T, Segawa M, Ogura K, Akagi T, Kubo K, Kaimori M and Funato T: WT1 mRNA level in peripheral blood is a sensitive biomarker for monitoring minimal residual disease in acute myeloid leukemia. Tohoku J Exp Med 219: 169-176, 2009.

15. Shibasaki Y, Seki Y, Tanaka T, Miyakoshi S, Fuse K, Kozakai T, Kobayashi H, Ushiki T, Abe T, Yano T, et al: The association of level of reduction of Wilms' tumor gene $1 \mathrm{mRNA}$ transcript in bone marrow and outcome in acute myeloid leukemia patients. Leuk Res 39: 667-671, 2015.

16. Marjanovic I, Karan-Djurasevic T, Ugrin M, Virijevic M, Vidovic A, Tomin D, Suvajdzic Vukovic N, Pavlovic S and Tosic N: Use of Wilms tumor 1 gene expression as a reliable marker for prognosis and minimal residual disease monitoring in acute myeloid leukemia with normal karyotype patients. Clin Lymphoma Myeloma Leuk 17: 312-319, 2017.

17. Elmaagacli AH, Beelen DW, Trenschel R and Schaefer UW: The detection of wt-1 transcripts is not associated with an increased leukemic relapse rate in patients with acute leukemia after allogeneic bone marrow or peripheral blood stem cell transplantation. Bone Marrow Transplant 25: 91-96, 2000.

18. Tamaki H, Mishima M, Kawakami M, Tsuboi A, Kim EH, Hosen N, Ikegame K, Murakami M, Fujioka T, Masuda T, et al: Monitoring minimal residual disease in leukemia using real-time quantitative polymerase chain reaction for Wilms tumor gene (WT1). Int J Hematol 78: 349-356, 2003.

19. Al-Mawali A, Gillis D and Lewis I: The use of receiver operating characteristic analysis for detection of minimal residual disease using five-color multiparameter flow cytometry in acute myeloid leukemia identifies patients with high risk of relapse. Cytometry Part B Clin Cytom 76: 91-101, 2009

20. Sun H, Li Y, Zhang ZF, Ju Y, Li L, Zhang BC and Liu B: Increase in myeloid-derived suppressor cells (MDSCs) associated with minimal residual disease(MRD)detection in adult acute myeloid leukemia. Int J Hematol 102: 579-586, 2015.

21. Ujj Z, Buglyó G, Udvardy M, Beyer D, Vargha G, Biró S and Rejtő L: WT1 expression in adult acute myeloid leukemia: Assessing its presence, magnitude and temporal changes as prognostic factors. Pathol Oncol Res 22: 217-221, 2016.

22. Di Grazia C, Pozzi S, Geroldi S, Grasso R, Miglino M, Colombo N, Tedone E, Luchetti S, Lamparelli T, Gualandi F, et al: Wilms tumor 1 expression and pre-emptive immunotherapy in patients with acute myeloid leukemia undergoing an allogeneic hemopoietic stem cell transplantation. Biol Blood Marrow Transplant 22: 1242-1246, 2016

23. Zhong L, Wei L, Chen J, Huang X, Gong Y and Lu Y: WT1 expression in circulating RNA as a minimal residual disease marker for AML patients after stem-cell transplantation. Mol Diagn Ther 19: 205-212, 2015.

24. Zhao XS, Yan CH, Liu DH, Xu LP, Liu YR, Liu KY, Qin YZ, Wang Y and Huang XJ: Combined use of WT1 and flow cytometry monitoring can promote sensitivity of predicting relapse after allogeneic HSCT without affecting specificity. Ann Hematol 92: 1111-1119, 2013.

(i) $\ominus$ This work is licensed under a Creative Commons Attribution-NonCommercial-NoDerivatives 4.0 International (CC BY-NC-ND 4.0) License. 